\title{
因HAD
}

DOI: http://doi.org/10.22585/hospdomic.v1i1.3

\section{Análisis bibliométrico de la producción científica, indizada en MEDLINE, sobre los servicios de salud proporcionados por las unidades de hospitalización a domicilio}

Bibliometric analysis of scientific production indexed in MEDINE, about hospital based home care services

Javier Sanz-Valero',2, Carmina Wanden-Berghe ${ }^{3}$

1. Universidad Miguel Hernández de Elche. Alicante, España.

2. Universidad de Alicante. Alicante, España.

3. Hospital General Universitario de Alicante, Instituto de Investigación Sanitaria y Biomédica de Alicante (ISABIAL-FISABIO). Alicante, España.

Correspondencia/Correspondence

isanz@umh.es

Recibido/Received

12.10.2016

Aceptado/Accepted

27.11.2016
Conflicto de Intereses/Competing interest Los autores declaran la no existencia de ningún tipo de conflicto de interés.

Financiación/Fundings

No declaran ninguna fuente específica de financiación ni ayuda económica recibida.

CÓMO CITAR ESTE TRABAJO | HOW TO CITE THIS PAPER

Sanz-Valero J, Wanden-Berghe C. Análisis bibliométrico de la producción científica, indizada en MEDLINE, sobre los servicios de salud proporcionados por las unidades de hospitalización a domicilio. Hosp Domic. 2017; 1 11):21-34. 


\section{RESUMEN}

Objetivo: Análisis bibliométrico y temático la producción científica relacionada con los servicios de salud proporcionados por las unidades de hospitalización a domicilio.

Método: Análisis bibliométrico. Los datos se obtuvieron de la base de datos MEDLINE, empleando el MeSH, «Home Care Services, Hospital-Based» como Major Topic. Fecha de la búsqueda: julio 2016. La muestra a estudio se calculó mediante estimación de parámetros poblacionales para una población infinita y la selección fue aleatoria simple sin reemplazo.

Resultados: Se analizaron 386 referencias. El número de originales fue de 204 (52,85\%), identificando 243 instituciones, con Índice de Cooperación de 3,75 $\pm 1,16$ autores/artículo. El idioma predominante fue el inglés con 279 (72,28\%) artículos. La obsolescencia, según el Índice de Burton-Kebler fue de 13 años y el Índice de Price del 14,40\%. El núcleo de Bradford lo constituyeron 23 revistas. La clasificación temática determinó una pertinencia del 70,73\%.

Conclusiones: Existencia de alta obsolescencia y orientación anglófona. Relación institucional e índice de corporación bajos. El acceso a la fuente primaria mejora a lo largo del tiempo, en línea a la iniciativa de acceso abierto, aunque con baja visibilidad. Los artículos estaban publicados de forma dispersa. La clasificación temática cumplió con la materia investigada.

Palabras clave: Servicios de atención a domicilio provisto por hospital; Bibliometría; Acceso a la Información; Bibliometría; Indicadores bibliométricos; Descriptores de ciencias de la salud.

\section{ABSTRACT}

Objective: A thematic and bibliometric analysis was done for the available scientific production about the home care services based in the hospital.

Methods: Bibliometric analysis. Data was obtained from MEDLINE database using MeSH "Home Care Services, Hospital-Based" as Major Topic. Search date: July 2016. The study sample was calculated by estimating population parameters for an infinite population and the selection was a simple random without replacement.

Results: A total of 386 references were analysed. The number of original articles was of 204 $(52,85 \%)$, identifying 243 institutions, with an index of cooperation of 3,75 $\pm 1,16$ authors/article. English was the predominant language in 279 $(72,28 \%)$ articles. The obsolescence was of 13 years according to the Burton-Kebler Index and the Price Index was of $14,40 \%$. Bradford nucleon was constituted by 23 journals. The thematic classification determines a relevance of $70.73 \%$.

Conclusions: There was a high obsolescence and an anglophone orientation. Also, there was a weak relation between institutions and corporation index. Over the time there was an improvement of the access to the primary source, in line with the Open Access initiative. The production was collected in a high number of journals (in a very dispersed form). The thematic classification meets the studied issue.

Keywords: Home care services, hospitalbased; Access to information; Bibliometrics; Bibliometric indicators; Medical subject headings. 


\section{INTRODUCCIÓN}

La publicación de un trabajo científico, es el modo más efectivo de transmitir un conocimiento obtenido como consecuencia de la investigación, y su visibilidad es importante para los propios investigadores, para las instituciones en que trabajan y las organizaciones que financian la investigación.

La investigación suele considerarse un indicador del grado de desarrollo. La investigación de un área como los servicios de salud proporcionados por las unidades de hospitalización a domicilio $(\mathrm{HaD})$, debería tener un impacto sobre la producción científica en consonancia con la importancia del problema, la capacidad investigadora y los recursos disponibles para generar dicha investigación (1).

El crecimiento de la producción científica en las últimas décadas y su indización en bases de datos bibliográficas automatizadas han potenciado el uso de la bibliometría y la generación de indicadores para medir los resultados de la actividad científica y tecnológica. El estudio empírico de la actividad científico-investigativa a partir de los artículos científicos publicados se basa en la aplicación de métodos cuantitativos, basados en indicadores y modelos matemáticos, que permiten caracterizar su desarrollo y evolución (2). Mediante los indicadores bibliométricos se pueden evaluar las revistas y otras publicaciones periódicas de manera objetiva, observar las tendencias que siguen y generar información útil para mejorar su gestión (3).

Como afirma Juárez-Rolando (4), la bibliometría es una herramienta útil para objetivar los productos de la investigación científica en las ciencias de la salud, volviendo tangibles procesos que permiten la toma de decisiones y la implementación de políticas de salud. Si bien, cuando se considera aisladamente, se podría argumentar que es altamente reduccionista, una puntuación cuantitativa, numérica, que no es representativa del rendimiento o la capacidad de un investigador. Sin embargo, la bibliometría tiene una gran utilidad, porque en términos pragmáticos representa una fuente de datos precisos y de obtención relativamente simple (5).

Por ello, el estudio de la producción científica en un área temática determinada, en este caso los servicios de salud proporcionados por las unidades de hospitalización a domicilio, es un buen indicador de la progresión de la investigación y de la generación de conocimientos sobre HaD. Su análisis bibliométrico permite un examen retrospectivo sobre el modo que ha sido investigada y dada a conocer, pero también puede evaluar el potencial de investigación de los grupos e instituciones involucradas (6).

En consecuencia, y teniendo en cuenta la edición de una revista específica sobre el Hospital a Domicilio, se ha creído adecuado realizar un estudio de su producción científica hasta el momento actual, año 2016, siendo el objetivo de este trabajo el analizar, mediante técnica bibliométrica, la literatura científica relacionada con los servicios de salud proporcionados por las unidades de hospitalización a domicilio indizada en MEDLINE.

\section{MÉTODOS}

\section{Diseño}

Estudio descriptivo trasversal de la producción científica sobre los servicios de salud proporcionados por las unidades de hospitalización a domicilio. 


\section{Fuente de obtención de los datos}

Se obtuvieron de la consulta directa y acceso, vía Internet, a esta literatura científica recogida en la base de datos MEDLINE, vía PubMed.

\section{Unidad de análisis}

Se trabajó con los artículos publicados y recuperados desde la base de datos bibliográfica indicada.

Para evitar posibles duplicidades en caso de publicación posterior como original u otro tipo documental, no se tuvieron en cuenta las comunicaciones a congresos, aunque sean de gran importancia para la comunidad investigadora ya que contribuyen a la rápida comunicación y difusión de la investigación.

\section{Búsqueda bibliográfica}

Para construir la ecuación de búsqueda se consultó el Medical Subject Headings (MeSH), Thesaurus desarrollado por la U.S. National Library of Medicine.

La ecuación empleada fue:

"Home Care Services, Hospital-Based"[Mesh] OR "Hospital-Based Home Care"[Title/ Abstract] OR "Hospital Based Home Care"[Title/Abstract] OR "Hospital Home Care Services"[Title/Abstract] OR "Hospital-Based Home Care Services"[Title/Abstract] OR "Hospital Based Home Care Services"[Title/Abstract] OR "Home Hospitalization"[Title/ Abstract] OR "Hospital at Home"[Title/Abstract] OR "Hospital-at-Home"[Title/Abstract] OR "Hospital Home Care"[Title/Abstract] OR "Hospital at Home Care"[Title/Abstract] OR "Hospital in the Home"[Title/Abstract].

La fecha de realización de la búsqueda fue julio de 2016.

\section{Cálculo del tamaño y método de muestreo}

El tamaño muestral se calculó mediante la estimación de parámetros poblacionales para una población infinita, (valor esperado $=0,5$; precisión del intervalo = 0,05; nivel de confianza $=0.95$ ). El tamaño muestral resultante fue de 386 referencias. Para la selección de las referencias a estudio se efectuó un muestreo aleatorio simple sin reemplazo, tomando como base el número total de las referencias bibliográficas obtenidas.

\section{Indicadores a estudio}

- Producción científica, calculada según número de artículos indizados.

- Tipología documental y número de artículos citables (suma de artículos de investigación original y de revisión).

- Índice de productividad (logaritmo del número de trabajos originales publicados).

- Edad: 2016 menos año de publicación del artículo.

- Semiperiodo de Burton-Kebler (Mediana de la distribución del conjunto de las referencias ordenadas por su antigüedad).

- Índice de Price (porcentaje de referencias con edad menor de 5 años). 
- Idioma de publicación del artículo

- Distribución geográfica de procedencia de los artículos.

- Índice de colaboración institucional: Número de instituciones que figuran como firmantes del documento.

- Filiación institucional del primer firmante e Índice de Lotka. Las instituciones se agruparon en tres niveles de rendimiento: pequeños productores (Índice de transitoriedad = un único trabajo), medianos productores (entre 2 y 9 trabajos) y grandes productores (10 o más trabajos).

- Número de autores por artículo e índice de colaboración (cociente entre el número de firmas y el número de trabajos).

- Revista donde se publica el artículo.

- Impacto de las publicaciones según el Journal Citation Report Science Edition Database (JCR), de la ISI Web of Science, Thomson Reuters y del SCImago Journal Rank (SJR) del SCImago Research Group de la Universidad de Granada.

- Dispersión: núcleo principal de Bradford (conjunto de revistas de mayor pertinencia para un área del conocimiento).

- Almetrías: medición del impacto de la investigación mediante la cuantificación de los enlaces, consultas y descargas del documento.

- Enlace: Existencia de enlace al documento desde PubMed.

- Acceso: Posibilidad de acceder al texto completo del artículo -gratuito o mediante pago- a través de del enlace existente en PubMed.

- DOI: digital object identifier, es un sistema de identificación (similar a los identificadores URI) que permite localizar, a través de la Red, el documento caracterizado mediante este código.

- Pertinencia temática de la producción científica: número de artículos indizados mediante el Descriptor (MeSH) "Home Care Services, Hospital-Based", como Major Topic.

\section{Análisis de los datos}

Las variables cualitativas se describieron por su frecuencia y relativa (porcentaje), las cuantitativas mediante su Media y Desviación Estándar, con Intervalos de Confianza del 95\%; representando las más relevantes mediante la utilización de tablas y gráficos. Se utilizó la Mediana, como medida de tendencia central, calculándose en ocasiones, el Máximo.

Para conocer la evolución de algunos indicadores se segmentó el período de estudio en dos etapas, tomando como punto de corte el índice de Burton-Kebler (la Mediana): $1^{a}$ época: de 1966 a 2002 y $2^{\mathrm{a}}$ época: de 2003 a 2016.

El crecimiento de la producción científica se examinó mediante el análisis de regresión. La existencia de asociación entre variables cualitativas se analizó mediante la prueba de chi-cuadrado de Pearson. Para comprobar la significación en la diferencia de medias para muestras independientes se utilizó la prueba t de Student. El nivel de significación utilizado en todos los contrastes de hipótesis fue $a \leq 0,05$.

Para el cálculo del tamaño muestral y la selección de las referencias del muestreo se utilizó el programa para análisis epidemiológico de datos tabulados EPIDAT versión 3.1.

Para el almacenamiento y análisis estadístico se utilizó el programa Statistical Package for the Social Sciences (IBM-SPSS), versión 22 para Windows.

El control de la calidad de la información se efectuó mediante la doble entrada de los datos, corrigiendo las inconsistencias mediante la consulta con los datos originales. 


\section{RESULTADOS}

\section{Producción científica}

De la consulta realizada en la base de datos MEDLINE se obtuvieron un total de 2131 referencias. Tras el cálculo del tamaño muestral para poblaciones infinitas se estudiaron 386 documentos.

El año más productivo fue el 2002 con 29 artículos, un 7,51 \% del total de la producción sobre los servicios de salud proporcionados por las unidades de HaD; IC95\% 4,88-10,14. El primer artículo indizado en la base de datos de referencia fue publicado en la revista British Medical Journal en mayo de 1894 (7).

La evolución de las publicaciones fue ascendente a lo largo de los años, ajustándose a un modelo lineal, dando un coeficiente de determinación $R 2=0,76$ y $p<0,001$, ver figura 1 .

Figura 1. Evolución anual de la producción científica sobre los servicios de salud proporcionados por las unidades de hospitalización a domicilio, recuperada de MEDLINE en julio de 2016, y ajuste al modelo lineal.

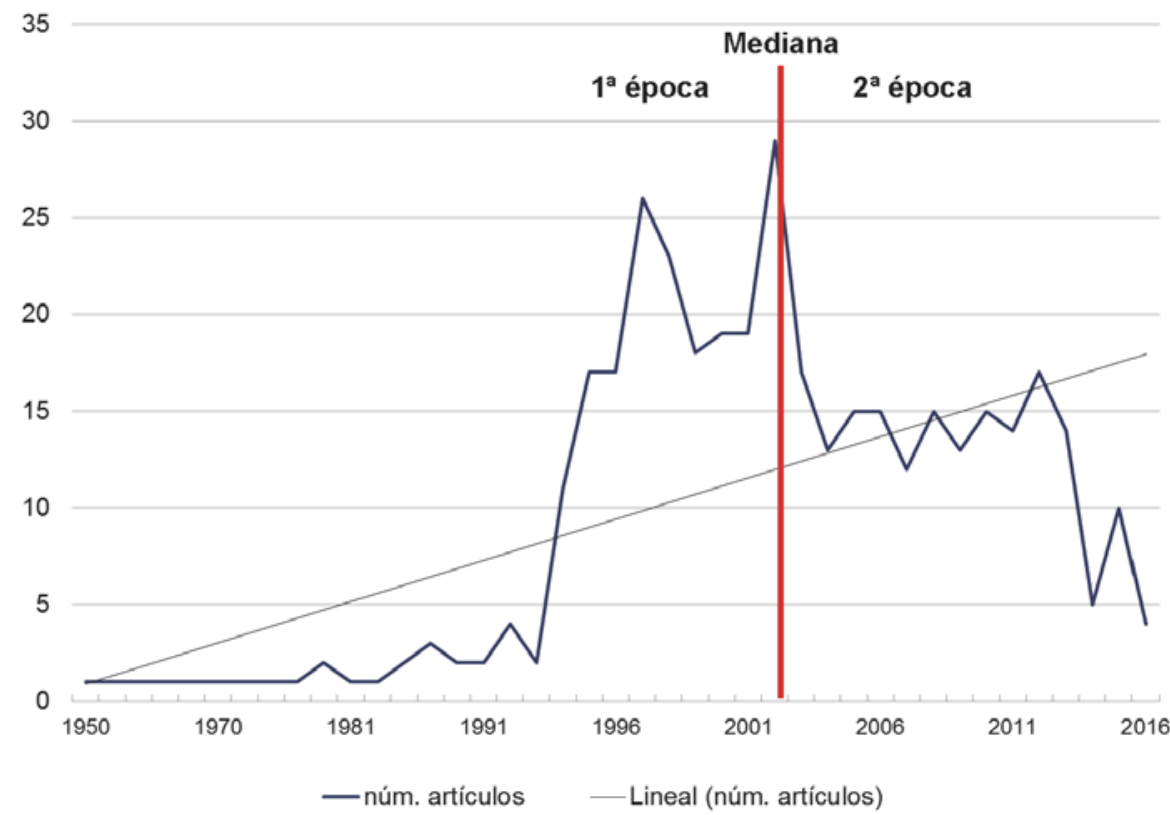

\section{Tipología documental}

La tipología documental más frecuente fue el artículo original con 204 documentos (52,85\%; IC95\% 47,87-57,83), el número de ensayos clínicos fue de 43 (11,14; IC95\% 8,00-14,28) y el de revisiones de 30 (7,77\%; IC95\% 5,10-10,44); ver tabla 1. El porcentaje de la suma de artículos de investigación original-incluyendo los ensayos clínicos- y de revisión, computables para el cálculo del impacto -los denominados artículos citables-, fue de 71,76\% (IC95\% 67,27-76,25). No se probaron 
diferencias significativas, relacionadas con la tipología documental citable, entre las dos épocas a estudio (chi cuadrado de Pearson $=4,33 ; \mathrm{gl}=2 ; \mathrm{p}=0,115$ ).

El índice de productividad general de artículos de investigación original fue de 2,31.

Tabla 1: Tipología documental de los artículos publicados sobre servicios de salud proporcionados por las unidades de hospitalización a domicilio, ordenados según época de estudio.

\begin{tabular}{|c|c|c|c|c|}
\hline \multirow[b]{2}{*}{ Tipo de artículo } & \multicolumn{2}{|c|}{$\begin{array}{l}1^{\text {a época }} \\
1966 \text { a } 2002\end{array}$} & \multicolumn{2}{|c|}{$\begin{array}{l}2^{a} \text { época } \\
2003 \text { a } 2016\end{array}$} \\
\hline & núm. & $\%$; IC $95 \%{ }^{a}$ & núm. & $\%$; IC $95 \%{ }^{b}$ \\
\hline Original & 107 & 27,$72 ; 23,25-32,19$ & 97 & 25,$13 ; 20,80-29,46$ \\
\hline Ensayo clínico & 17 & 4,$40 ; 2,36-6,45$ & 26 & 6,$74 ; 4,24-9,24$ \\
\hline Revisión & 11 & 2,$85 ; 1,19-4,51$ & 19 & 4,$92 ; 2,76-7,08$ \\
\hline Carta & 10 & 2,$59 ; 1,01-4,18$ & 8 & 2,$07 ; 0,65-3,49$ \\
\hline Opinión & 25 & 6,$48 ; 4,02-8,93$ & 7 & 1,$81 ; 0,48-3,14$ \\
\hline Editorial & 6 & 1,$55 ; 0,32-2,79$ & 5 & 1,$30 ; 0,17-2,42$ \\
\hline Noticias & 2 & 0,$52 ; 0,00-1,23$ & 4 & 1,$04 ; 0,03-2,05$ \\
\hline Serie de casos & 20 & 5,$18 ; 2,97-7,39$ & 6 & 1,$55 ; 0,32-2,79$ \\
\hline Comentario & 2 & 0,$52 ; 0,00-1,23$ & 7 & 1,$81 ; 0,48-3,14$ \\
\hline Entrevista & 2 & 0,$52 ; 0,00-1,23$ & -- & --- \\
\hline Guía clínica & 5 & 1,$30 ; 0,17-2,42$ & -- & --- \\
\hline
\end{tabular}

\section{Edad de los artículos y obsolescencia/actualidad de la producción científica}

La edad media de las referencias analizadas, fue de 12,60 \pm 0,42 años (IC95\% 11,77-13,42), con mediana de 13 (Semiperiodo de Burton-Kebler) y máximo 52 años. El índice de Price calculado fue del $14,40 \%$.

\section{Idioma, procedencia geográfica y filiación institucional}

El idioma predominante de las referencias recuperadas fue el inglés, con 279 casos $(72,28 \%$; IC95\% 67,81-76,75). Dejar constancia del resto de idiomas utilizados en más de 10 referencias estudiadas: japonés 38 (9,84\%; IC95\% 6,87-12,82), francés 19 (4,92\%; IC95\% 2,76-7,08), español 17 (4,40\%; IC95\% 2,36-6,45) y alemán con 13 referencias (3,37\%; IC95\% 1,57-5,17).

No se obtuvieron diferencias significativas relacionadas con el idioma entre las dos épocas a estudio (chi cuadrado de Pearson $=18,74 ; g l=13 ; p=0,131$ ).

En el conjunto de búsqueda se identificaron documentos de 34 nacionalidades distintas, siendo Estados Unidos de América el país con mayor producción con 59 referencias (15,28\%; IC95\% $11,70-18,87)$. El resto de países, ordenados según época, pueden consultarse en la tabla 2, encontrándose diferencias significativas entre las dos épocas a estudio (chi cuadrado de Pearson = 63,56; $\mathrm{gl}=34 ; \mathrm{p}=0$ 0,002). En 47 artículos (12,18\%; 8,91-15,44) no constaba el país de procedencia.

Se identificaron trabajos publicados de 243 instituciones, con una Mediana de 1 centro por artículo y Máximo de 21. La media del número de instituciones por artículo -índice de colaboración institucional- fue de $1,56 \pm 0,11$, siendo significativamente mayor en la $2^{\mathrm{a}}$ época $(1,91 \pm 0,19)$ que en la $1^{\mathrm{a}}(1,16 \pm 0,48) ; \mathrm{p}<0,001$, ver tabla 3 . Un total de 58 estudios $(15,03 \%$; IC95\% 11,46-18,59) fueron realizados en colaboración entre 2 o más centros. 
Tabla 2: Distribución por países de los trabajos publicados sobre servicios de salud proporcionados por las unidades de hospitalización a domicilioa, ordenados según época de estudio.

\begin{tabular}{|c|c|c|c|c|}
\hline \multirow[b]{2}{*}{ País } & \multicolumn{2}{|c|}{$\begin{array}{l}1^{a} \text { época } \\
1966 \text { a } 2002\end{array}$} & \multicolumn{2}{|c|}{$\begin{array}{l}2^{a} \text { época } \\
2003 \text { a } 2016\end{array}$} \\
\hline & núm. & $\% ;$ IC95\% ${ }^{\mathrm{b}}$ & núm. & $\% ;$ IC95\% ${ }^{\mathrm{b}}$ \\
\hline Estado Unidos & 31 & 8,$03 ; 5,32-10,74$ & 28 & 7,$25 ; 4,67-9,84$ \\
\hline Japón & 28 & 7,$25 ; 4,67-9,84$ & 13 & 3,$37 ; 1,57-5,17$ \\
\hline Reino Unido & 21 & 5,$44 ; 3,18-7,70$ & 24 & 6,$22 ; 3,81-8,63$ \\
\hline Francia & 16 & 4,$15 ; 2,16-6,13$ & 11 & 2,$85 ; 1,19-4,54$ \\
\hline Australia & 13 & 3,$37 ; 1,57-5,17$ & 15 & 3,$89 ; 1,96-5,81$ \\
\hline Suecia & 12 & 3,$11 ; 1,38-4,84$ & 8 & 2,$07 ; 0,65-3,49$ \\
\hline España & 9 & 2,$33 ; 0,83-3,84$ & 14 & 3,$63 ; 1,76-5,49$ \\
\hline Canadá & 7 & 1,$81 ; 0,48-3,14$ & 7 & 1,$81 ; 0,48-3,14$ \\
\hline Alemania & 6 & 1,$55 ; 0,32-2,79$ & 6 & 1,$55 ; 0,32-2,79$ \\
\hline Italia & 3 & 0,$78 ; 0,00-1,65$ & 9 & 2,$33 ; 0,83-3,84$ \\
\hline
\end{tabular}

Tabla 3: Distribución de las instituciones productoras de más de 2 trabajos publicados sobre servicios de salud proporcionados por las unidades de hospitalización a domicilio, ordenados por época de estudio.

\begin{tabular}{|c|c|c|c|c|}
\hline \multirow[b]{2}{*}{ Institución } & \multicolumn{2}{|c|}{$\begin{array}{l}1^{a} \text { época } \\
1966 \text { a } 2002\end{array}$} & \multicolumn{2}{|c|}{$\begin{array}{l}2^{a} \text { época } \\
2003 \text { a } 2016\end{array}$} \\
\hline & núm. & $\% ;$ IC95\% ${ }^{b}$ & núm. & $\% ; I^{C} 95 \%{ }^{b}$ \\
\hline Karolinska Institut & 3 & 0,$78 ; 0,00-1,65$ & 3 & 0,$78 ; 0,00-1,65$ \\
\hline John Hopkins Institutions & 1 & 0,$26 ; 0,00-0,77$ & 3 & 0,$78 ; 0,00-1,65$ \\
\hline Royal Melbourne Hospital & 1 & 0,$26 ; 0,00-0,77$ & 3 & 0,$78 ; 0,00-1,65$ \\
\hline Linköping University & 2 & 0,$52 ; 0,00-1,23$ & 2 & 0,$52 ; 0,00-1,23$ \\
\hline Kaname-cho Hospital & 4 & 1,$04 ; 0,03-2,05$ & - & --- \\
\hline Bristol University & 4 & 1,$04 ; 0,03-2,05$ & - & --- \\
\hline Royal Brompton Hospital & - & --- & 3 & 0,$78 ; 0,00-1,65$ \\
\hline Prince of Wales Hospital & 2 & 0,$52 ; 0,00-1,23$ & 1 & 0,$26 ; 0,00-0,77$ \\
\hline Pennsylvania University & 2 & 0,$52 ; 0,00-1,23$ & 1 & 0,$26 ; 0,00-0,77$ \\
\hline
\end{tabular}

Las instituciones se clasificaron en tres niveles de rendimiento extrapolando a las mismas el Índice de Lotka: índice de transitoriedad: 207 (53,63\%; IC95\% 48,65-58,60) eran pequeños productores ( 1 trabajo publicado), 36 (9,33\%; IC95\% 6,43-12,23) eran medianos productores (entre 2 y 10 trabajos) y no se observó ninguna institución con más de 10 trabajos. En 89 artículos (23,06; IC95\% 18,86-27,26) no figuraba ninguna institución de filiación.

\section{Autoría}

Se contabilizaron un total de 1397 autores, con un máximo de 20 autores observado en un solo artículo. La media, equivalente al Índice de Cooperación, fue de 3,75 $\pm 1,16$ autores (IC95\% $3,43-4,06)$. La mediana fue de 3 autores por artículo. En la $2^{\circ}$ época a estudio, la media de autores/ artículo fue superior a la observada en la $1^{a}$ época: 4,59 versus 2,99, $p<0,001$. El mismo hecho fue observado cuando se calculó para los artículos citables: 5,08 versus 3,457, $p<0,001$. 
La evolución del número de autores por artículo fue ascendente a lo largo de los años, ajustándose a un modelo de curva exponencial con un coeficiente de determinación $R 2=0,15$ y $p<0,001$.

\section{Revistas, impacto y dispersión de la literatura científica}

A partir de la muestra seleccionada se recuperaron un total de 237 revistas, de las que 23 componían el núcleo principal de Bradford, ver figura 2. Como puede observarse en la tabla 4, las revistas que publicaron 5 o más artículos sobre el tema a estudio fueron 9.

Figura 2: Dispersión de las revistas y los artículos sobre servicios de salud proporcionados por las unidades de hospitalización a domicilio en la base de datos bibliográfica MEDLINE (julio 2016).

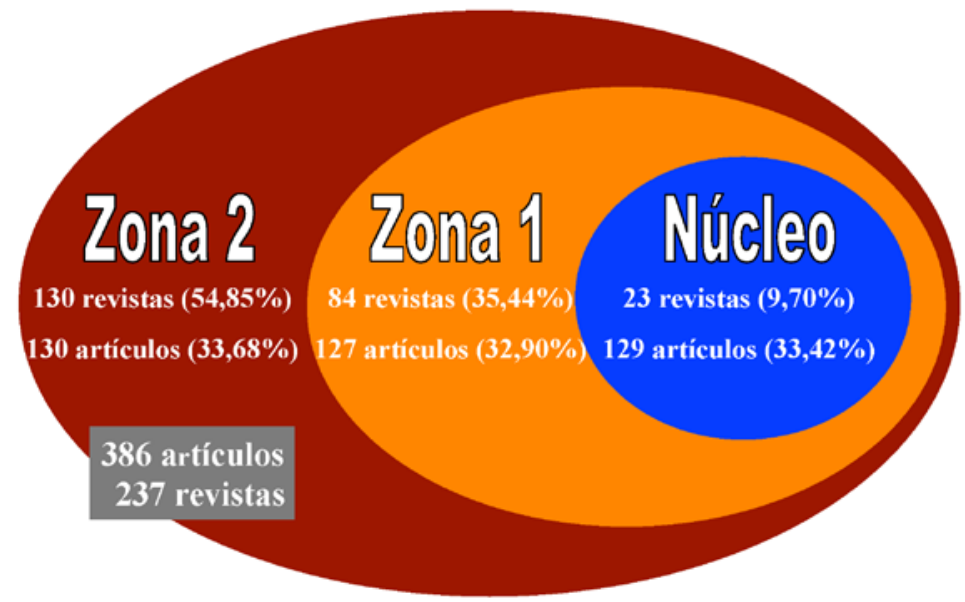

Tabla 4. Revistas que han publicado 5 o más artículos sobre servicios de salud proporcionados por las unidades de hospitalización a domicilio.

\begin{tabular}{|c|c|c|c|c|c|c|}
\hline Abreviatura de la Revista & $f_{0}$ & $\%$ & $\mathrm{FI}_{(\mathrm{JCR})}$ & $\mathbf{Q}_{(\mathrm{JCR})}$ & SJR & $Q_{(\text {SJR) }}$ \\
\hline Gan To Kagaku Ryoho & 34 & 14,35 & --- & --- & -- & -- \\
\hline Med Clin (Barc) & 8 & 3,38 & 1,267 & 2 & 0,221 & 3 \\
\hline Home Healthc Nurse & 8 & 3,38 & --- & -- & 0,160 & 3 \\
\hline Med J Aust & 6 & 2,53 & 3,369 & 1 & 1,029 & 1 \\
\hline BMJ & 6 & 2,53 & 19,697 & 1 & 2,567 & 1 \\
\hline Soins & 5 & 2,11 & --- & --- & 0,101 & 4 \\
\hline Caring & 5 & 2,11 & --- & --- & 0,100 & 4 \\
\hline Palliat Med & 5 & 2,11 & 3,685 & 1 & 1,914 & 1 \\
\hline J Am Geriatr Soc & 5 & 2,11 & 3,842 & 1 & 2,236 & 1 \\
\hline \multicolumn{7}{|c|}{$\begin{array}{l}\mathrm{f}_{0}=\text { Número de artículos publicados; FI(JCR) = Factor de Impacto según la base de datos Journal Citation } \\
\text { Report para 2015; Q(JCR) = Cuartil según la mejor posición que ocupa la revista en cualquier categoría en } \\
\text { la base de datos Journal Citation Report; SJR = Indicador Scimago Journal Rank para 2015; Q(SJR) = Cuart } \\
\text { según la mejor posición que ocupa la revista en cualquier categoría en la base de datos Scimago Journal \& } \\
\text { Country Rank. }\end{array}$} \\
\hline
\end{tabular}


El estudio de la dispersión de la literatura científica recuperada, determina la concentración de una frecuencia similar de documentos en un número diferente de revistas: núcleo principal, con 23 revistas $(9,70 \%)$ que incluyen 129 artículos (33,42\%). El núcleo 2 con 84 revistas $(35,44 \%)$ que contienen 127 artículos (32,90\%) y el núcleo 3 con 130 revistas (54,85\%) que engloban 130 artículos (33,68\%); ver figura 2: gráfico de dispersión según Bradford.

La medición del impacto de estas publicaciones mediante técnicas altmétricas se pudo observar en 63 documentos (16,32\%), siendo significativo el mayor uso en la $2^{\text {a }}$ época a estudio -45 artículos versus 18- (chi cuadrado de Pearson $=19,01 ; \mathrm{gl}=1 ; \mathrm{p}<0,001$ ).

\section{Acceso al documento primario}

En relación al enlace al texto completo desde MEDLINE se comprobó que se pudo acceder a él en 191 ocasiones (49,48\%) siendo en 75 ocasiones (19,43\%) de forma gratuita. Existieron diferencias significativas, a favor de la $2^{a}$ época, tanto en el enlace al documento (chi cuadrado de Pearson $=86,00 ; \mathrm{gl}=1 ; \mathrm{p}<0,001$ ), como a la consulta del texto de forma gratuita (chi cuadrado de Pearson $=6,95 ; g l=1 ; p=0,01)$.

Se constató que 156 documentos (40,41\%) estaban identificados mediante DOI, observando diferencias a favor de la $2^{\mathrm{a}}$ época $(65,92 \%$ versus $18,36 \%$; chi cuadrado de Pearson $=90,18 ; \mathrm{gl}=$ $1 ; p<0,001)$.

\section{Clasificación temática de la producción científica}

Para conocer la pertinencia temática de la producción científica a estudio se comprobó que de los 386 documentos seleccionados como muestra a estudio, 273 (70,73\%) de ellos estaban indizados mediante el MeSH "Home Care Services, Hospital-Based", como Major Topic. Comprobándose la no existencia de diferencias, en cuanto a la indización mediante este Descriptor, entre las dos épocas a estudio (chi cuadrado de Pearson $=1,471 ; g l=1 ; p=0,226$ ).

\section{DISCUSIÓN}

El presente estudio ofrece, en base a los principales indicadores bibliométricos, una visión retrospectiva sobre la producción científica relacionada con los servicios de salud proporcionados por las unidades de HaD. El estudio temático mediante el Descriptor como Major Topic informó que los documentos recuperados estaban en consonancia con la temática seleccionada.

En esta producción se observó un progresivo incremento de los documentos publicados, que bien es cierto no logró un modelo de crecimiento exponencial. Hay algunas explicaciones a este hecho: que esta progresión se obtiene en periodos muy superiores a los 30 años y sobre todo, que en esta área temática y según los volúmenes de producción, no se ha alcanzado la llamada «explosión de la información». Así, el año más productivo fue el 2002 y, a partir de él y contrariamente a lo esperado, se produjo un descenso en la generación de artículos (8).

El predominio de los artículos originales era esperado al tratarse de un área de estudio emergente con eminentemente aplicación empírica. El porcentaje de artículos citables se debe considerar insuficiente, los indicadores de evaluación sugieren una proporción mínima del 50\% (3). Por otro lado, no es de extrañar la presencia de ensayos clínicos al tratarse de un área de estudio con aplicación clínica (9). 
El análisis de la obsolescencia, medido tanto por el índice de Burton-Kleber como por el Índice de Price, mostraron resultados superiores a lo previsto en el área de las ciencias de la salud (10). Esta menor actualidad de los indicadores se explica también por el descenso de la productividad a partir del año 2002.

La procedencia mayoritaria de artículos con filiación anglófona, especialmente estadounidense, es un hecho conocido y ya recogido en la literatura científica (11). Un claro ejemplo de esta superioridad en cuanto a la producción científica, es que de las referencias añadidas a la base de datos MEDLINE en el periodo 2005-2009, cerca del 45\% fueron artículos publicados en los Estados Unidos (12).

Igualmente, se conoce que el predominio del inglés es una constante en las revistas de las ciencias de la salud. El idioma de publicación es importante por cuanto se ha comprobado que, cualquiera sea la naturaleza de un artículo científico, las posibilidades de recibir citaciones son mayores si se publica en inglés $(13,14)$.

No se localizaron artículos de grandes grupos de investigación -con más de 10 publicaciones sobre la materia- lo que puede lastrar la visibilidad y el impacto de la materia estudiada (11). Es positivo, que en la segunda época a estudio existiera una mayor colaboración institucional con respecto a la primera, pero con valores por debajo a lo esperado (15). En esta línea, sería interesante conocer el presupuesto que se invierte en Investigación y Desarrollo (I+D) en la temática a estudio. Quizá este dato nos diera un índice ajustado de la producción de cada institución en esta categoría del conocimiento (1).

El número de autores y el índice de cooperación presentaron datos similares a los de otras revistas del ámbito de las ciencias de la salud (10). Es conveniente señalar que los datos analizados no mostraron inclinación hacia las autorías colectivas, lo cual indica una baja colaboración entre autores y en la formación de grupos de trabajo, elementos indispensables para el desarrollo de la ciencia. Ahora bien, la leve evolución en el tiempo del número de autores por artículo es un dato alentador, aunque mejorable (16).

Las revistas que contienen un mayor número de artículos sobre $\mathrm{HaD}$ no presentan un enfoque exclusivo en la temática, si se exceptúa a la revista Home Healthcare Nurse, lo que puede explicar a su vez la amplia dispersión que se ha observado en este estudio. Hubiera sido esperable que un pequeño grupo de revistas -apenas una docena- concentrara un alto número de artículos. La presencia de publicaciones en revistas de alto impacto, indizadas en las principales bases de datos bibliográficas, como British Medical Journal o Journal of the American Geriatrics Society, es lógico dado el tipo de pacientes que ingresan en $\mathrm{HaD}$ y la búsqueda de la mayor visibilidad. Como queda representado en la tabla 4 y en la figura 2 existe una manifiesta variabilidad en relación al impacto de las revistas donde se han publicado los artículos analizados. De todos modos, la falta de concentración de los artículos puede deberse a la carencia de revistas específicas de la especialidad.

La implementación de indicadores altimétricos en estas publicaciones es aún escasa (menos de 2 de cada 10 artículos). No obstante, se observó un significativo aumento en la época más actual. En todo caso, hay que intentar mejorar los valores obtenidos, debido a que estas herramientas en línea tienen un gran potencial sobre el impacto de los datos de la investigación y pueden funcionar como complemento de otro tipo de indicadores $(17,18)$.

El acceso al documento primario queda claro que se ve auxiliado mediante el uso de Internet. Existiendo, lógicamente, una mayor accesibilidad on-line a partir del año 2000 por la gran eclosión de las bases de datos bibliográficas, hecho que se constata en este trabajo al comprobarse diferencias significativas, en relación al enlace al texto completo del artículo, en el periodo más reciente (2a época) (9).

En cuanto al acceso al texto completo de esta literatura científica, se prueba un escaso apoyo a la iniciativa Open Access (menos de 1 de cada 20 artículos analizados), muy probablemente por 
no existir publicaciones de referencia que faciliten el acceso libre, directo y gratuito a la publicación científica sobre HaD.

Es significativo la presencia, y aumento en el tiempo, de la utilización del Digital Object Identifier (DOI) ya que este identificador, similar a un identificador de recursos uniforme (URI), permite a los investigadores un acceso rápido y persistente al documento primario (19). Lo que es patente, es que su implantación y uso es, hoy por hoy, incuestionable (20). Por ende, que más de la mitad de los artículos estudiados en la segunda época tuviera DOI, facilita la recuperación de los artículos sobre HaD.

La utilización del Descriptor MeSH (en este caso, como Major Topic) sirvió para analizar los trabajos indizados mediante este Descriptor y proporcionado la posibilidad de clasificación temática que no hubiera sido posible tan sólo a través del título o del resumen del trabajo. Los resultados sobre la clasificación temática de la producción científica muestran unos datos altamente relacionados con el área de conocimiento analizada, representada por el descriptor «Home Care Services, Hospital-Based» e indican un excelente porcentaje, en cuanto a la indización, en las dos épocas analizadas (21). La introducción de este Descriptor, en el tesauro de la U.S. National Library of Medicine, permite realizar adecuadamente la recuperación de esta documentación y demuestra la adecuación de la ecuación de búsqueda empleada para la consecución de esta información de manera precisa.

Posibles limitaciones de este trabajo. Si bien, se ha utilizado el autor designado para la correspondencia para estudiar la filiación institucional y esto puede ir en detrimento del resto de los autores del artículo, y ser un posible sesgo, existen trabajos que han analizado la no existencia de diferencias significativas entre tomar solo este autor o la totalidad de ellos (9). No se estudió la relación entre el número de autores por artículo y el número de citas ya que trabajos previos refieren una débil relación (22) o incluso la no existencia de diferencias significativas (23).

Por todo lo anteriormente expuesto, se puede concluir: Los indicadores métricos obtenidos están en línea con otras áreas de conocimiento relacionadas con las ciencias de la salud. Si bien, los resultados sobre obsolescencia fueron superiores a lo esperado. La producción científica presentó un incremento lineal, ofreciendo mejores indicadores en la $2^{a}$ época a estudio. Los autores de los trabajos sobre $\mathrm{HaD}$ deberían apostar por un mayor apoyo a la iniciativa Open Access (mayor visibilidad). La clasificación temática mostró datos altamente relacionados con el área de conocimiento analizada.

\section{BIBLIOGRAFÍA}

1. Wanden-Berghe C, Martín-Rodero H. 25 años de investigación en nutrición y alimentación en el espacio iberoamericano del conocimiento. Nutr Hosp. 2012;27(Supl 2):26-33. DOI: 10.3305/ nh.2012.27.sup2.6270; PMID: 23568394

2. Allen L, Jones C, Dolby K, Lynn D, Walport M. Looking for landmarks: the role of expert review and bibliometric analysis in evaluating scientific publication outputs. PloS One. 2009;4(6):e5910. DOI: 10.1371/journal.pone.0005910; PMID: 19536339

3. Sanz-Valero J, Casterá VT, Wanden-Berghe C. Estudio bibliométrico de la producción científica publicada por la Revista Panamericana de Salud Pública. Rev Panam Salud Pública. 2014;35(2):81-8. PMID: 24781088

4. Juárez-Rolando P. Bibliometría para la evaluación de la actividad científica en ciencias de la salud. Rev Enferm Hered. 2016;9(1):57-61. DOI: 10.20453/renh.v9i1.2864 
5. Davidson PM, Newton PJ, Ferguson C, Daly J, Elliott D, Homer C, et al. Rating and ranking the role of bibliometrics and webometrics in nursing and midwifery. Scientific World $\mathrm{J}$. 2014;2014:135812. DOI: 10.1155/2014/135812; PMID: 24550691

6. Sanz-Valero J, Tomás-Gorriz V, Morales-Suárez-Varela M. Estudio bibliométrico de la producción científica publicada por la revista Ars Pharmaceutica en el periodo 2001 a 2013. Ars Pharmaceurica. 2014;55(2):1-10.

7. A Hospital at Home. Br Med J. 1894;1(1742):1102. PMID: 20754828

8. Ardanuy J. Breve introducción a la bibliometría. Barcelona, España: Universidad de Barcelona; 2012.

9. Sanz-Valero J, Gil Á, Wanden-Berghe C, Martínez de Victoria E; Grupo CDC-Nut SENPE. Análisis bibliométrico y temático de la producción científica sobre ácidos grasos omega-3 indizada en las bases de datos internacionales sobre ciencias de la salud. Nutr Hosp. 2012;27(Supl 2):41-8. DOI: 10.3305/nh.2012.27.sup2.6272; PMID: 23568396

10. Tomás-Casterá V, Sanz-Valero J, Juan-Quilis V. Estudio bibliométrico de la producción científica y de consumo de las revistas sobre nutrición indizadas en la red SciELO. Nutr Hosp. 2013;28(3):969-70. DOI: 10.3305/nh.2013.28.3.6463; PMID: 23848128

11. Arnett JJ. The neglected $95 \%$ : why American psychology needs to become less American. Am Psychol. 2008;63(7):602-14. DOI: 10.1037/0003-066X.63.7.602; PMID: 18855491

12. Franco-Pérez AM, Sanz-Valero J, Wanden-Berghe C, Melian-Fleitas L. La producción científica iberoamericana en ciencias de la nutrición: La indización en PubMed y Google Scholar. Nutr Hosp. 2014;30(5):1165-72. DOI: 10.3305/nh.2014.30.5.7723; PMID: 25365023

13. Valdés $S \mathrm{G}$, Pérez G F, Reyes $B \mathrm{H}$. Análisis de las publicaciones biomédicas chilenas indizadas en PubMed, en los años 2008 y 2009. Rev Médica Chile. 2015;143(8):979-86. DOI: 10.4067/ S0034-98872015000800003; PMID: 26436925

14. Franco-López A, Sanz-Valero J, Culebras J. Publicar en castellano, o en cualquier otro idioma que no sea inglés, negativo para el factor de impacto y citaciones. JONNPR. 2016;1(2):65-70. DOI: 10.19230/jonnpr.2016.1.2.1005

15. Sanz-Valero J, Tomás-Casterá V, Tomás-Gorriz V. Estudio bibliométrico de producción y consumo de la revista Farmacia Hospitalaria (2004-2012). Farm Hosp. 2014;38(1):1-8. DOI: 10.7399/ FH.2014.38.1.1153; PMID: 24483853

16. Camargo Jr KR, Coeli CM. Multiple authorship: growth or inflationary bubble? Rev Saúde Pública. 2012;46(5):894-900. PMID: 23128267

17. Haustein S, Peters I, Bar-Ilan J, Priem J, Shema H, Terliesner J. Coverage and adoption of altmetrics sources in the bibliometric community. Scientometrics. 2014;101(2):1145-63. DOI: $10.1007 / \mathrm{s} 11192-013-1221-3$

18. Haustein S, Costas R, Larivière V. Characterizing social media metrics of scholarly papers: The effect of document properties and collaboration patterns. PLoS ONE. 2015;10(3):e0120495. DOI: 10.1371/journal.pone.0120495

19. DeRisi S, Kennison R, Twyman N. The what and whys of DOls. PLoS Biol. 2003;1(2):e57. DOI: 10.1371/journal.pbio.0000057; PMID: 14624257

20. Brase J, Lautenschlager M, Sens I. The tenth anniversary of assigning DOI names to scientific data and a five year history of DataCite. D-Lib Mag. 2015;21(1-2). DOI: 10.1045/january2015brase 
Artículo Original: Sanz-Valero et al.

21. Guardiola-Wanden-Berghe R, Sanz-Valero J, Wanden-Berghe C. Medical subject headings versus American Psychological Association Index Terms: indexing eating disorders. Scientometrics. 2013;94(1):305-11. DOI: 10.1007/s11192-012-0866-7

22. Lemu R, Koricheva J. Does scientific collaboration increase the impact of ecological articles? Bioscience. 2005;55(5):438-43. DOI: 10.1641/0006-3568(2005)055[0438:DSCITI]2.0.CO;2

23. Hart R. Collaboration and article quality in the literature of academic librarianship. J Acad Libr. 2007;33(2):190-5. DOI: 10.1016/j.acalib.2006.12.002Ute. 